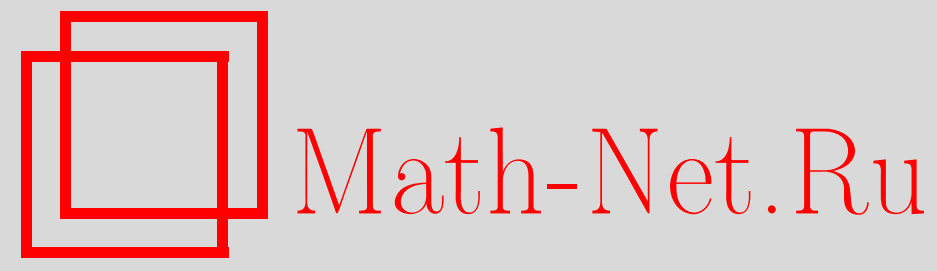

С. А. Назаров, Критерий существования затухающих решений в задаче о резонаторе с цилиндрическим волноводом, Функи. анализ и его прил., 2006, том 40, выпуск 2, 20-32

DOI: https://doi.org/10.4213/faa4

Использование Общероссийского математического портала Math-Net.Ru подразумевает, что вы прочитали и согласны с пользовательским соглашением

http://www. mathnet.ru/rus/agreement

Параметры загрузки:

IP : 54.197 .130 .99

26 апреля 2023 г., 03:31:06 


\title{
Критерий существования затухающих решений в задаче о резонаторе с цилиндрическим волноводом*
}

\author{
(C) 2006. C. A. НАЗАРОВ
}

\section{§1. Постановка задачи}

1. Задача о резонаторе с цилиндрическим волноводом. Пусть $\Omega$ область в пространстве $\mathbb{R}^{n}$ с гладкой границей $\partial \Omega$, совпадающая внутри полупространства $\mathbb{R}_{+}^{n}=\left\{x=(y, z): y=\left(x_{1}, \ldots, x_{n-1}\right) \in \mathbb{R}^{n-1}, z=x_{n}>0\right\}$ с цилиндром $Q=\{x: y \in \omega, z \in \mathbb{R}\}$ (волноводом); здесь $\omega$ - область в $\mathbb{R}^{n-1}$, ограниченная гладкой замкнутой поверхностью $\partial \omega$. Считаем, что подмножество $G=\{x \in \Omega: z<0\}$ (резонатор) имеет компактное замыкание $\bar{G}=G \cup \partial G$. В области $\Omega$ рассмотрим задачу Дирихле для уравнения Гельмгольца

$$
\begin{aligned}
\Delta_{x} u(x)+k^{2} u(x) & =0, & & x \in \Omega, \\
u(x) & =0, & & x \in \partial \Omega .
\end{aligned}
$$

Как известно, задачу (1.1) нужно дополнить подходящими условиями излучения на бесконечности. Пусть $\lambda_{n}$ и $\varphi_{n}-$ собственные числа и отвечающие им собственные функции задачи Дирихле для оператора Лапласа $-\Delta_{y}$ на сечении $\omega$, причем

$$
\begin{gathered}
0<\lambda_{1}<\lambda_{2} \leqslant \lambda_{3} \leqslant \cdots \leqslant \lambda_{n} \leqslant \cdots \rightarrow+\infty, \\
\left(\varphi_{n}, \varphi_{m}\right)_{\omega}=\delta_{n, m}, \quad n, m \in \mathbb{N}:=\{1,2, \ldots\},
\end{gathered}
$$

где $\delta_{n, m}$ - символ Кронекера, а $(,)_{\omega}-$ скалярное произведение в пространстве $L_{2}(\omega)$. Предположим сначала, что $\lambda_{n} \neq k^{2}$ при всех $n \in \mathbb{N}$, и введем обозначения

$$
\begin{gathered}
U_{n}^{ \pm}(x)=\left(2\left|\Lambda_{n}\right|\right)^{-1 / 2} \exp \left(\mp i \Lambda_{n} z\right) \varphi_{n}(y), \\
\Lambda_{n}=\left(k^{2}-\lambda_{n}\right)^{1 / 2}, \quad \operatorname{Re} \Lambda_{n} \geqslant 0, \operatorname{Im} \Lambda_{n} \geqslant 0 .
\end{gathered}
$$

При $n \in N_{-}=\left\{m \in \mathbb{N}: \lambda_{m}<k^{2}\right\}$ формула (1.3) задает осииллирующие волны в цилиндре $Q$; в случае $n \in N_{+}=\left\{m \in \mathbb{N}: \lambda_{m}>k^{2}\right\}$ волна $U_{n}^{-}$экспоненциально затухает, а волна $U_{n}^{+}$экспоненциально возрастает при $z \rightarrow+\infty$. Положим $N_{+}=$ $\{\boldsymbol{n}+1, \boldsymbol{n}+2, \ldots\}$.

Если имеется собственное значение $\lambda_{\boldsymbol{n}-\varkappa+1}=\cdots=\lambda_{\boldsymbol{n}}=k^{2}$ ненулевой кратности $\varkappa$ (такая ситуация называется пороговой), то $\Lambda_{\boldsymbol{n}-\varkappa+1}=\cdots=\Lambda_{\boldsymbol{n}}=0$, а вместо волн вида ${ }^{1)}(1.3)_{1}$ для соответствующих значений $n$ имеются пары волн

$$
U_{n}^{0}(x)=\varphi_{n}(y), \quad U_{n}^{1}(x)=z \varphi_{n}(y), \quad n=\boldsymbol{n}-\varkappa+1, \ldots, \boldsymbol{n} .
$$

*Работа выполнена при финансовой поддержке Российского фонда фундаментальных исследований (проект 04-01-00567).

1) Нижний индекс указывает номер строки в соответствующей формуле. 
При этом $N_{-}=\{1, \ldots, \boldsymbol{n}-\varkappa\}$. Положим также $N_{0}=N_{-} \cup\{\boldsymbol{n}-\varkappa+1, \ldots, \boldsymbol{n}\}$. Разумеется, величины $\boldsymbol{n}, \varkappa$ и множества $N_{+}, N_{-}, N_{0}$ зависят от $k$ и $\omega$, но это не отражено в обозначениях. Заметим, что $N_{0} \neq N_{-}$только в пороговой ситуации.

При отсутствии пороговых собственных значений физические условия излучения, отвечающие прибытию волны $U_{p}^{+}$с бесконечности и ее отражению резонатором $G$, выглядят так:

$$
u_{p}(x)=U_{p}^{+}(x)+\sum_{q=1}^{n} s_{p q} U_{q}^{-}(x)+O\left(e^{-z \operatorname{Im} \Lambda_{n+1}}\right), \quad p=1, \ldots, \boldsymbol{n} .
$$

Набор $\left\{u_{1}, \ldots, u_{\boldsymbol{n}}\right\}$ решений задачи (1.1), (1.5) определяет $\boldsymbol{n} \times \boldsymbol{n}$-матрицу рассеяния $s=\left(s_{p q}\right)$, которая оказывается унитарной (см., например, [1]).

В пороговой ситуации к совокупности уходящих волн $U_{1}^{-}, \ldots, U_{\boldsymbol{n}}^{-}$ж обычно присоединяют «стоячие» волны $U_{\boldsymbol{n}-\varkappa+1}^{0}, \ldots, U_{\boldsymbol{n}}^{0}$ из списка $(1.4)$ (частный случай осциллирующих), однако получающиеся таким образом физические условия излучения не позволяют определить унитарную матрицу рассеяния размера $\boldsymbol{n} \times \boldsymbol{n}$. Дело в том, что осциллирующие волны $U_{1}^{ \pm}, \ldots, U_{n-\varkappa}^{ \pm}$подчинены условиям

$$
\boldsymbol{q}\left(U_{m}^{ \pm}, U_{n}^{ \pm}\right)= \pm i \delta_{m, n}, \quad \boldsymbol{q}\left(U_{m}^{ \pm}, U_{n}^{\mp}\right)=0,
$$

обеспечивающим унитарность матрицы рассеяния, но $\boldsymbol{q}\left(U_{n}^{0}, U_{n}^{0}\right)=0$ при $n=$ $\boldsymbol{n}-\varkappa+1, \ldots, \boldsymbol{n}$. В левых частях соотношений (1.6) расположена антисимметричная полуторалинейная форма

$$
\boldsymbol{q}(u, v)=\int_{\omega}\left(u(y, 0) \overline{\partial_{z} v(y, 0)}-\overline{v(y, 0)} \partial_{z} u(y, 0)\right) d y .
$$

Подчеркнем, что для волн $(1.3)_{1}$ и (1.4), удовлетворяющих задаче (1.1) в цилиндре $Q$, интеграл (1.7), возникающий в формуле Грина, не зависит от выбора сечения, т. е. в равной мере аргументами функций из подынтегрального выражения (1.7) могут служить $y, t$ при любом $t \in \mathbb{R}$.

В статье [2] (см. также $\$ 5.6$ книги [3]) замечено, что для линейных комбинаций волн (1.4)

$$
U_{n}^{ \pm}(x)=2^{-1 / 2}\left(U_{n}^{0}(y) \mp i U_{n}^{1}(y, z)\right), \quad n=\boldsymbol{n}-\varkappa+1, \ldots, \boldsymbol{n},
$$

соотношения (1.6) также оказываются верными, а значит, матрица $s$ коэффициентов из представлений (1.5) с волнами (1.3) 1 и (1.8) вновь становится унитарной (см., например, [2]). Условия излучения (1.5), включающие линейно растущие волны, названы естественными.

2. «Ловушечные моды». Физически осмысленным является поиск чисел $k \in \mathbb{R}_{+}$, при которых задача (1.1) имеет нетривиальные решения, исчезающие на бесконечности, - по понятной причине они называются ловушечными модами. При помощи метода Фурье можно убедиться в том, что упомянутые решения убывают с экспоненциальной скоростью и образуют конечномерный линеал $\mathfrak{D}$ (это верно и для общих эллиптических краевых задач; см., например, гл. 3 и 5 в [3]). K настоящему времени разными методами построено множество примеров затухающих решений для различных краевых условий и форм области $\Omega$ (см. [4-12] и др.). В [13] для задачи о падении плоской волны на периодическую границу и в [14] для общих самосопряженных эллиптических задач 
разработана процедура, оперирующая с расширенной матрицей рассеяния (см. далее разд. 2 §3) и позволяющая определить число линейно независимых решений, которые убывают не быстрее $O\left(e^{-\beta z}\right)$ при заданном наперед показателе $\beta>0$. Использованные в $[13,14]$ конструкции, первоначально имевшие отношение к постановке условий Соболева на ребрах границы (см. [15]), не допускают предельного перехода $\beta \rightarrow+\infty$, и поэтому вопрос об идентификации точечного спектра оператора задачи (1.1), рассматриваемого как неограниченный в $L_{2}(\Omega)$, тем не менее остался открытым. Подчеркнем, что элементы подпространства $\mathfrak{D} \subset L_{2}(\Omega)$ суть собственные функции этого оператора.

В настоящей статье строится унитарный оператор $\mathfrak{S}$ «фиктивного рассеяния» и устанавливается биективное отображение линеала $\mathfrak{D}$ на подпространство собственных векторов оператора $\mathfrak{S}_{+}$, отвечающих собственному числу 1 (теорема 3.1). Под $\mathfrak{S}_{+}$подразумевается сужение оператора $\mathfrak{S}$ на ортогональное дополнение в $L_{2}(\omega)$ линейной оболочки функций $\varphi_{1}, \ldots, \varphi_{\boldsymbol{n}}$. Матрица рассеяния $s$ и все расширенные матрицы рассеяния связываются с конечномерными аппроксимациями оператора $\mathfrak{S}$ (разд. $2 \S 3$ ). Отметим, что оператор $\mathfrak{S}$ определяется по решениям смешанной краевой задачи в ограниченной области $G$ и алгорифм его построения допускает численную реализацию.

\section{§2. Оператор фиктивного рассеяния}

1. Оператор Стеклова-Пуанкаре. Собственные функции $\varphi_{n}$ образуют ортонормированный базис в пространстве $L_{2}(\omega)$. Введем оператор

$$
\sum_{n=1}^{\infty} a_{n} \varphi_{n}=\varphi \longmapsto T \varphi=\sum_{n=1}^{\infty} i\left(\Lambda_{n}+\chi(n)\right) a_{n} \varphi_{n},
$$

где $\chi(n)=1$ при $n=\boldsymbol{n}-\varkappa+1, \ldots, \boldsymbol{n}$ и $\chi(n)=0$ для остальных целых $n$, т. е. вне порога слагаемое $\chi(n)$ в правой части (2.1) отсутствует. Поскольку $\left|\Lambda_{n}\right|=O\left(\lambda_{n}^{1 / 2}\right)$ в силу определения (1.3), отображение $T: \stackrel{\circ}{H}{ }^{1}(\omega) \rightarrow L_{2}(\omega)$ непрерывно; здесь $\stackrel{\circ}{H}^{1}(\omega)$ - подпространство функций из соболевского класса $H^{1}(\omega)$, обращающихся в нуль на $\partial \omega$. Выполняется ортогональное разложение

$$
L_{2}(\omega)=\mathscr{L}_{0} \oplus \mathscr{L}_{+},
$$

в котором $\mathscr{L}_{0}$ - линейная оболочка собственных функций $\varphi_{n}$ с индексами $n \in N_{0}$. Ортогональные проекторы на подпространства $\mathscr{L}_{0}$ и $\mathscr{L}_{+}$обозначим символами $\mathscr{P}_{0}$ и $\mathscr{P}_{+}$. Форма

$$
(T \varphi, \psi)_{\omega}=i \sum_{n=1}^{\infty}\left(\Lambda_{n}+\chi(n)\right) a_{n} \bar{b}_{n},
$$

где $a_{n}$ и $b_{n}$ - коэффициенты разложений функций $\varphi$ и $\psi$ по базису $\left\{\varphi_{n}\right\}$, определена по замыканию на пространстве $H_{00}^{1 / 2}(\omega)$ с нормой

$$
\left\|\mid \varphi ; H_{00}^{1 / 2}(\omega)\right\| \|=\left(\left\|\varphi ; H^{1 / 2}(\omega)\right\|^{2}+\left\|\rho^{-1 / 2} \varphi ; L_{2}(\omega)\right\|^{2}\right)^{1 / 2}
$$

(см. 1.11 книги [16]), где $H^{1 / 2}(\omega)$ - пространство Соболева-Слободецкого, а $\rho(y)=\operatorname{dist}(y, \partial \omega)$. Подчеркнем, что структура нормы $(2.3)$ проистекает из условий Дирихле на $\partial \omega$ для собственных функций $\varphi_{n}$. В частности, следы на сечении $\Upsilon=\omega \times\{0\}$ функций из подпространства $\stackrel{\circ}{H}{ }^{1}(\Omega ; \Gamma)$ (аннулируется след на 
части $\Gamma=\partial \Omega \cap \partial G$ границы резонатора) попадают именно в $H_{00}^{1 / 2}(\omega)$. Поскольку $-i \Lambda_{n}>0$ при $n \in N_{+}$и $\Lambda_{n}>0$ при $n \in N_{-}$, равенство $(T \varphi, \varphi)_{\omega}=0$ имеет место только в случае $\varphi=0$. Итак, $T$ можно рассматривать как непрерывный оператор в гильбертовом пространстве $\mathscr{H}=H_{00}^{1 / 2}(\omega)$. Его сужения $T_{0}$ и $T_{+}$на подпространства $\mathscr{H}_{0}=\mathscr{P}_{0} \mathscr{H}$ и $\mathscr{H}_{+}=\mathscr{P}_{+} \mathscr{H}$ являются кососимметрическим и симметрическим операторами соответственно; кроме того, операторы $i T_{0}$ и $T_{+}$ отрицательно определены.

При помощи метода Фурье нетрудно убедиться в том, что $T$ - оператор Стеклова-Пуанкаре, сопоставляющий данным Дирихле $\Phi$ нормальную производную $\partial_{z} V(y, 0)$ решения $V$ задачи

$$
\begin{gathered}
\Delta_{x} V(x)+k^{2} V(x)=0, \quad x \in Q_{+}=\omega \times \mathbb{R}_{+}, \\
V(x)=0, \quad x \in(\partial Q)_{+}=\partial \omega \times \mathbb{R}_{+}, \quad V(y, 0)=\Phi(y), \quad y \in \omega,
\end{gathered}
$$

снабженной естественными (физическими вне порога) условиями излучения

$$
V(x)-\sum_{n=1}^{n} a_{n} U_{n}^{-}(x)=O\left(e^{-z \operatorname{Im} \Lambda_{n+1}}\right) .
$$

Коэффициенты $a_{n}$, конечно же, самими условиями (2.5) не фиксируются.

Иными словами, для (единственного) решения задачи (2.4), (2.5) справедливо равенство $\partial_{z} V(y, 0)=T \Phi(y)$. В частности, благодаря соотношениям

$$
\begin{aligned}
& \partial_{z} U_{n}^{ \pm}(y, 0)-T U_{n}^{ \pm}(y, 0)=-\left(2\left|\Lambda_{n}\right|\right)^{-1 / 2} i \Lambda_{n}(1 \pm 1) \varphi_{n}(y), \quad n \in N_{-} \cup N_{+}, \\
& \partial_{z} U_{n}^{ \pm}(y, 0)-T U_{n}^{ \pm}(y, 0)=-2^{-1 / 2} i(1 \pm 1) \varphi_{n}(y), \quad n=\boldsymbol{n}-\varkappa+1, \ldots, \boldsymbol{n},
\end{aligned}
$$

задача (1.1), (1.5) в неограниченной области $\Omega$ эквивалентна задаче в ограниченной области $G$

$$
\begin{gathered}
\Delta_{x} w_{p}(x)+k^{2} w_{p}(x)=0, \quad x \in G, \quad w_{p}(x)=0, \quad x \in \Gamma=\partial \Omega \cap \mathbb{R}_{-}^{n}, \\
\partial_{z} w_{p}(y, 0)-T w_{p}(y, 0)=g_{p}(y), \quad x \in \Upsilon=\omega \times\{0\},
\end{gathered}
$$

в которой правая часть имеет вид

$g_{n}(y)=-i\left(2 \Lambda_{n}\right)^{1 / 2} \varphi_{n}(y), \quad n \in N_{-}, \quad g_{n}(y)=-i 2^{1 / 2} \varphi_{n}(y), \quad n=\boldsymbol{n}-\varkappa+1, \ldots, \boldsymbol{n}$.

Разрешимость задачи обеспечивается следующим утверждением, которое, в частности, гарантирует включение $w_{p} \in C^{\infty}(\bar{G})$.

Лемма 2.1. Сужение на множество $G$ любого (гладкого) решения $u_{p}$ задачи (1.1), (1.5) удовлетворяет задаче (2.7) с правой частъю из (2.8), а обобщенное решение $w_{p} \in \stackrel{o}{H}^{1}(G ; \Gamma)$ последней задачи, восполненное на полуцилиндре $Q_{+}$по формулам

$$
\begin{gathered}
v_{p}(x)=U_{p}^{+}(x)+2^{1 / 2} \sum_{n=1}^{\infty}\left(\left|\Lambda_{n}\right|+\chi(n)\right)^{1 / 2} b_{p n} U_{n}^{-}(x), \\
b_{p n}=a_{p n}-\left[2\left(\left|\Lambda_{n}\right|+\chi(n)\right)\right]^{-1 / 2} \delta_{p, n}, \quad a_{p n}=\left(w_{p} \mid \Upsilon, \varphi_{n}\right)_{\omega},
\end{gathered}
$$

является гладким на замыкании $\bar{\Omega}$ и удовлетворяет задаче (1.1), (1.5).

ДоказАтельство. Утверждение о решениях $u_{p}$ обеспечено выкладками (2.6). Из формул (2.9) следует, что функция $v_{p} \in \stackrel{\circ}{H}_{\mathrm{loc}}^{1}\left(\bar{Q}_{+} ; \partial Q \backslash \bar{\Upsilon}\right)$ является 
гладкой при $z>0$. Кроме того, она подчинена условиям излучения (1.5) и равенствам $(2.4)$ с $\Phi(y)=w_{p}(y, 0)$. В силу формул $(2.1)$ и $(2.7)-(2.9)$ имеем

$$
\begin{aligned}
\partial_{z} w_{p} \mid \Upsilon & =g_{p}+T w_{p} \mid \Upsilon=-i\left[2\left(\Lambda_{p}+\chi(p)\right)\right]^{1 / 2} \varphi_{p}+i \sum_{n=1}^{\infty}\left(\Lambda_{n}+\chi(n)\right) a_{n} \varphi_{n} \\
& =-i 2^{-1 / 2}\left(\Lambda_{p}+\chi(p)\right)^{1 / 2} \varphi_{p}+i \sum_{n=1}^{\infty}\left(\Lambda_{n}+\chi(n)\right) b_{n} \varphi_{n}=\partial_{z} v_{p} \mid \Upsilon, \quad p=1, \ldots, \boldsymbol{n} .
\end{aligned}
$$

Фигурирующие здесь ряды заведомо сходятся в $H_{00}^{-1 / 2}(\omega)$, однако, так как $w_{p}$ и $\partial_{z} w_{p}$ совпадают с $v_{p}$ и $\partial_{z} v_{p}$ на сечении $\Upsilon$, функция, равная $w_{p}$ на $G$ и $v_{p}$ на $Q_{+}$, становится обобщенным решением задачи (1.1) и поэтому приобретает бесконечную дифференцируемость всюду в $\bar{\Omega}$ благодаря эллиптичности задачи.

Поскольку $u_{p}=v_{p}=w_{p}$ на $\Upsilon$, соотношения (2.9), (1.5) и $(1.2)_{2}$ влекут за собой следующие интегральные представления элементов матрицы рассеяния:

$$
s_{p q}=-\delta_{p, q}+\left[2\left(\Lambda_{q}+\chi(q)\right)\right]^{1 / 2} \int_{\omega} w_{p}(y, 0) \varphi_{q}(y) d y, \quad p, q=1, \ldots, \boldsymbol{n} .
$$

Отсюда вытекает, что даже при наличии нетривиальных затухающих решений задачи (1.1), а значит, и нетривиальных решений однородной задачи (2.7) матрица рассеяния $s$ определена однозначно: следы на сечении $\Upsilon$ упомянутых решений ортогональны в $L_{2}(\omega)$ функциям $\varphi_{1}, \ldots, \varphi_{\boldsymbol{n}}$.

2. Симметризация оператора Стеклова-Пуанкаре. Изменив в определении (2.1) коэффициенты при функциях $\varphi_{\boldsymbol{n}+1}, \varphi_{\boldsymbol{n}+2}, \ldots$, отвечающих экспоненциальным волнам, преобразуем оператор (2.1) в кососимметрический:

$$
\mathscr{T}=T \mathscr{P}_{0}-i T \mathscr{P}_{+} .
$$

Симметрический оператор $\mathscr{M}=-i \mathscr{T}$ действует по формуле

$$
\sum_{n=1}^{\infty} a_{n} \varphi_{n}=\varphi \longmapsto \mathscr{M} \varphi=\sum_{n=1}^{\infty}\left(\left|\Lambda_{n}\right|+\chi(n)\right) a_{n} \varphi_{n}
$$

и является положительно определенным. Положительный квадратный корень из $\mathscr{M}$ обозначаем символом $\mathscr{M}^{1 / 2}$; он осуществляет изоморфизмы $H_{00}^{1 / 2}(\omega) \approx$ $L_{2}(\omega)$ и $L_{2}(\omega) \approx H_{00}^{-1 / 2}(\omega)$, где $H_{00}^{-1 / 2}(\omega)$ - пространство, двойственное к $H_{00}^{1 / 2}(\omega)$. Обращаем внимание на легко проверяемые равенства

$$
\mathscr{P}_{0} \mathscr{M}=-i \mathscr{P}_{0} T, \quad \mathscr{P}_{+} \mathscr{M}=-\mathscr{P}_{+} T .
$$

Очевидно, что проекторы $\mathscr{P}_{0}$ и $\mathscr{P}_{+}$коммутируют с операторами $T$ и $\mathscr{T}, \mathscr{M}$. Если рассматривать $T$ как оператор в гильбертовом пространстве $H_{00}^{1 / 2}(\omega)$ (см. комментарий к определению $(2.3))$, то $\mathscr{M}=\left(T^{*} T\right)^{1 / 2}$.

Рассмотрим аналогичную (2.7) задачу

$$
\begin{gathered}
\Delta_{x} w(x)+k^{2} w(x)=0, \quad x \in G, \quad w(x)=0, \quad x \in \Gamma, \\
\partial_{z} w(y, 0)-\mathscr{T} w(y, 0)=g(y), \quad x \in \Upsilon=\partial G \backslash \bar{\Gamma},
\end{gathered}
$$


в которой $g \in H_{00}^{-1 / 2}(\omega)$. Под обобщенным решением задачи (2.14) подразумеваем элемент $w$ пространства $\mathfrak{H}:=\stackrel{\circ}{H}{ }^{1}(G ; \Gamma)$, удовлетворяющий при любой пробной функции $v \in \mathfrak{H}$ интегральному тождеству

$$
\left(\nabla_{x} w, \nabla_{x} v\right)_{G}-k^{2}(w, v)_{G}-i(\mathscr{M} w, v)_{\Upsilon}=(g, v)_{\Upsilon},
$$

где $(,)_{G}$ - скалярное произведение в $L_{2}(G)$, а $(,)_{\Upsilon}$ - расширение скалярного произведения в $L_{2}(\Upsilon)$ до двойственности между пространствами $\mathscr{H}=$ $H_{00}^{1 / 2}(\Upsilon)$ и $\mathscr{H}^{*}=H_{00}^{-1 / 2}(\Upsilon)$.

ЛЕмма 2.2. При любой функиии $g \in \mathscr{H}^{*}$ задача $(2.15)=(2.14)$ имеет единственное обобщенное решение $w \in \mathfrak{H}$.

ДокАзАтельство. Согласно теореме Рисса о представлении непрерывного функционала в гильбертовом пространстве, переформулируем тождество (2.15)) как абстрактное уравнение

$$
w+(K+i M) w=\mathfrak{g} \in \mathfrak{H} .
$$

При этом $K$ и $M$ - непрерывные и симметрические операторы в пространстве $\mathfrak{H}=\stackrel{\circ}{H}^{1}(G ; \Gamma)$, снабженном скалярным произведением $\left(\nabla_{x} w, \nabla_{x} v\right)_{G}$, а правая часть $\mathfrak{g} \in \mathfrak{H}$ определена так, что $\left(\nabla_{x} v, \nabla_{x} \mathfrak{g}\right)_{G}=(v, g)_{\Upsilon}$ для любого $v \in \mathfrak{H}$. Оператор $K$ компактный, т. е. по альтернативе Фредгольма достаточно убедиться в отсутствии нетривиальных решений $w^{ \pm} \in \mathfrak{H}$ однородных уравнений

$$
w^{ \pm}+K w^{ \pm} \pm i M w^{ \pm}=0 .
$$

Если $w^{ \pm}$- такое решение, то, учитывая эквивалентность формулировок $(2.15)$ и (2.16), получаем равенство

$$
\left(\nabla_{x} w^{ \pm}, \nabla_{x} w^{ \pm}\right)_{G}-k^{2}\left(w^{ \pm}, w^{ \pm}\right)_{G}= \pm i\left(\mathscr{M} w^{ \pm}, w^{ \pm}\right)_{\Upsilon} .
$$

Поскольку левая часть вещественна, $\left(\mathscr{M} w^{ \pm}, w^{ \pm}\right)_{\Upsilon}=0$, т. е. $w^{ \pm} \mid \Upsilon=0$ благодаря положительной определенности оператора (2.12). Следовательно, функция $w^{ \pm}$ принадлежит пространству $\stackrel{\circ}{H}$ ( $(G)$ и удовлетворяет интегральному тождеству

$$
\left(\nabla_{x} w^{ \pm}, \nabla_{x} v\right)_{G}=k^{2}\left(w^{ \pm}, v\right)_{G} \quad \forall v \in \stackrel{\circ}{H}^{1}(G ; \Gamma) .
$$

Иными словами, $w^{ \pm}-$собственная функция смешанной краевой задачи для оператора Лапласа. Как известно (см., например, [17]), нетривиальная собственная функция не может полностью аннулироваться на множестве $\Upsilon$, где заданы условия Неймана. Полученное противоречие доказывает, что уравнения (2.17) имеют лишь тривиальные решения.

3. Унитарный оператор фиктивного рассеяния. Пусть $\varphi \in L_{2}(\omega)$, а $\mathscr{W}(\varphi) \in \stackrel{\circ}{H}^{1}(G ; \Gamma)-$ решение задачи $(2.15)$ с функцией

$$
g=-2^{1 / 2} i \mathscr{M}^{1 / 2} \varphi
$$

в правой части. Определим оператор $\mathfrak{S}$ в пространстве $L_{2}(\omega)$ по формуле

$$
\mathfrak{S} \varphi=i \varphi-\left.2^{1 / 2} i \mathscr{M}^{1 / 2} \mathscr{W}(\varphi)\right|_{\Upsilon}
$$

Подчеркнем, что в случае $\varphi=\varphi_{n}$, где $n=1, \ldots, \boldsymbol{n}$, функции $(2.18)$ и $(2.8)$ совпадают. Поскольку

$$
\left\|\mathscr{W}(\varphi) ; H_{00}^{1 / 2}(\Upsilon)\right\| \leqslant c\left\|\mathscr{W}(\varphi) ; H^{1}(G)\right\| \leqslant c\left\|g ; H_{00}^{-1 / 2}(\omega)\right\| \leqslant c\left\|\varphi ; L_{2}(\omega)\right\|,
$$


оператор $\mathfrak{S}$ непрерывен. Проверим его изометричность.

Пусть $\varphi, \psi \in L_{2}(\omega)$. Напишем дважды тождество (2.15), положив один раз $w=\mathscr{W}(\varphi), v=\mathscr{W}(\psi)$, а другой $w=\mathscr{W}(\psi), v=\mathscr{W}(\varphi)$. Второе тождество после комплексного сопряжения вычтем из первого. Формула (2.18) обеспечивает равенство

$$
0=i\left(\mathscr{M} \mathscr{W}(\varphi)-2^{1 / 2} \mathscr{M}^{1 / 2} \varphi, \mathscr{W}(\varphi)\right)_{\Upsilon}+i\left(\mathscr{W}(\varphi), \mathscr{M} \mathscr{W}(\psi)-2^{1 / 2} \mathscr{M}^{1 / 2} \psi\right)_{\Upsilon}
$$

Продолжим преобразование правой части при помощи соотношения (2.19):

$$
\begin{aligned}
0= & \frac{i}{2}\left(2^{1 / 2} i \mathscr{M}^{1 / 2} \mathscr{W}(\varphi)-2 i \varphi, 2^{1 / 2} i \mathscr{M}^{1 / 2} \mathscr{W}(\psi)\right)_{\Upsilon} \\
& +\frac{i}{2}\left(2^{1 / 2} i \mathscr{M}^{1 / 2} \mathscr{W}(\varphi), 2^{1 / 2} i \mathscr{M}^{1 / 2} \mathscr{W}(\psi)-2 i \psi\right)_{\Upsilon} \\
= & \frac{i}{2}\left((-\mathfrak{S} \varphi-i \varphi,-\mathfrak{S} \psi+i \psi)_{\Upsilon}+(-\mathfrak{S} \varphi+i \varphi,-\mathfrak{S} \psi-i \psi)_{\Upsilon}\right) \\
= & i(\mathfrak{S} \varphi, \mathfrak{S} \psi)_{\Upsilon}-i(\varphi, \psi)_{\Upsilon} .
\end{aligned}
$$

Таким образом, оператор $\mathfrak{S}$ изометричен.

Teорема 2.1. $\mathfrak{S}$ - унитарный оператор в $L_{2}(\omega)$.

ДокАЗАТЕЛЬСтво. Осталось убедиться в сюръективности оператора $\mathfrak{S}$. Пусть $\Psi$ - произвольный элемент пространства $L_{2}(\Omega)$, а $V \in \stackrel{\circ}{H^{1}}(G ; \Gamma)$ - peшение задачи $(2.15)$, в которой краевое условие на сечении $\Upsilon$ заменено таким:

$$
\partial_{z} V(y, 0)+\mathscr{T} V(y, 0)=2^{1 / 2} \mathscr{M}^{1 / 2} \Psi(y), \quad y \in \omega .
$$

В лемме 2.2 было показано, что видоизмененная задача также однозначно разрешима. Положим $\varphi=-i \Psi-2^{1 / 2} \mathscr{M}^{1 / 2} V \in L_{2}(\omega)$; тогда выполняется равенство $\left.\partial_{z} V\right|_{\Upsilon}-\left.\mathscr{T} V\right|_{\Upsilon}=2^{1 / 2} i \mathscr{M}^{1 / 2} \varphi$, т. е. $V=-\mathscr{W}(\varphi)$ в силу определения $(2.18)$. Теперь соотношение (2.19) принимает вид

$$
\mathfrak{S} \varphi=i\left(-i \Psi-2^{1 / 2} \mathscr{M}^{1 / 2} V\right)-2^{1 / 2} i \mathscr{M}^{1 / 2} \mathscr{W}(\varphi)=\Psi
$$

что и требовалось.

Унитарный оператор $\mathfrak{S}$ назовем оператором фиктивного рассеяния - причины выбора термина поясняются в разд. $2 \S 3$. Отметим, что формула (2.19) весьма похожа на определение характеристического оператора узла (см. $[18$, $\S 1.3]$ ), но содержит «лишний» множитель $i$, а именно, характеристический оператор в [18] совпадает с матрицей рассеяния $s$ (см. далее следствие 3.1). Однако на этом и кончаются аналогии с книгой [18], в которой подпространство $\operatorname{ker}\left\{s-\mathbb{I}_{0}\right\}$ собственных векторов матрицы рассеяния $s$, отвечающих собственному числу 1 , ассоциируется с собственными внутренними состояниями узла и возможностью закрытия каналов рассеяния. Постановка искусственных краевых условий (2.14) $)_{2}$ в некотором не связанном с определениями из [18] смысле «открывает» все возможные каналы рассеяния, а в финальной формуле (3.12) фигурирует собственное число $-i$ матрицы рассеяния $s$. В частности, последнее обстоятельство создает впечатление ненатуральности «рассеяния», порождающего оператор $\mathfrak{S}$, который далее послужит для описания необходимых и достаточных условий существования экспоненциально затухающих решений задачи (1.1). 


\section{§3. Критерий существования ловушечных мод}

1. Основная теорема. Цель этого раздела - установить взаимно однозначное соответствие между подпространством $\mathfrak{D}$ экспоненциально затухающих решений однородной задачи $(1.1)$ и линеалом $\operatorname{ker}\left(\mathfrak{S}_{+}-\mathbb{I}_{+}\right)$собственных функций оператора $\mathfrak{S}_{+}=\mathscr{P}_{+} \mathfrak{S} \mathscr{P}_{+}$в $\mathscr{L}_{+}$, отвечающих собственному числу $1 ;$ здесь $\mathbb{I}_{+}-$ тождественное отображение в $\mathscr{L}_{+}$, а $\mathscr{L}_{+}$- компонента ортогонального разложения (2.2).

Теорема 3.1. Отображсение

$$
L_{2}(\omega)=\mathfrak{D} \ni u \longmapsto \mathfrak{N} u=\varphi:=\left.2^{-1 / 2}(1-i) \mathscr{M}^{1 / 2} u\right|_{\Upsilon}
$$

является взаимно однозначным отображением на линеал $\operatorname{ker}\left\{\mathfrak{S}_{+}-\mathbb{I}_{+}\right\} \subset$ $\mathscr{L}_{+} \subset L_{2}(\omega)$.

ДокАЗАТЕльСТво. Ввиду экспоненциального затухания решение $u \in \stackrel{\circ}{H}^{1}(\Omega)$, суженное на полуцилиндр $Q_{+}$, допускает разложение

$$
\left.u\right|_{Q_{+}}=\sum_{n=\boldsymbol{n}+1}^{\infty} a_{n} U_{n}^{-} .
$$

Таким образом, $\left.u\right|_{\Upsilon} \in \mathscr{H}_{+}$и $\varphi \in \mathscr{M}^{1 / 2} \mathscr{H}_{+}=\mathscr{L}_{+}$. Кроме того, используя формулы (2.13) и (3.1), заключаем, что $\left.u\right|_{G}$ - решение задачи $(2.14)$ с правой частью

$$
g=\left.\partial_{z} u\right|_{\Upsilon}-\left.\mathscr{T} u\right|_{\Upsilon}=-\left.\mathscr{M} u\right|_{\Upsilon}-\left.i \mathscr{M} u\right|_{\Upsilon}=-2^{1 / 2} i \mathscr{M}^{1 / 2} \varphi .
$$

Сравнивая это равенство с определением (2.18), видим, что $\mathscr{W}(\varphi)=\left.u\right|_{G}$. Следовательно, согласно формулам (3.1) и (2.19) выполняется соотношение

$$
\mathfrak{S} \varphi=i \varphi-\left.2^{1 / 2} i \mathscr{M}^{1 / 2} u\right|_{\Upsilon}=i \varphi-i(1+i) \varphi=\varphi,
$$

означающее, в частности, что $\mathscr{P}_{+} \mathfrak{S} \varphi=\varphi$. Иными словами, образ $\mathfrak{N D}$ содержится в ядре $\operatorname{ker}\left\{\mathfrak{S}_{+}-\mathbb{I}_{+}\right\}$. Отображение (3.1) инъективно, так как при $\left.u\right|_{\Upsilon}=0$ разложение (3.2) влечет за собой невозможное равенство $\left.u\right|_{Q_{+}}=0$.

Для проверки сюръективности построим обратный оператор $\mathfrak{N}^{-1}: \operatorname{ker}\left\{\mathfrak{S}_{+}-\right.$ $\left.\mathbb{I}_{+}\right\} \rightarrow \mathfrak{L}$. Прежде всего заметим, что, учитывая изометричность оператора $\mathfrak{S}$, при $\varphi \in \operatorname{ker}\left\{\mathfrak{S}_{+}-\mathbb{I}_{+}\right\}$имеем

$$
\|\varphi\|^{2}=\|\mathfrak{S} \varphi\|^{2}=\left\|\mathscr{P}_{+} \mathfrak{S} \varphi\right\|^{2}+\left\|\mathscr{P}_{0} \mathfrak{S} \varphi\right\|^{2} \Longrightarrow \mathscr{P}_{0} \mathfrak{S} \varphi=0,
$$

т. е. $\mathfrak{S} \varphi=\varphi$. При учете соотношений $(2.14)_{2}$ и (2.18), (2.19) находим, что

$$
\begin{aligned}
\left.\partial_{z} \mathscr{W}(\varphi)\right|_{\Upsilon} & =\left.i \mathscr{M} \mathscr{W}(\varphi)\right|_{\Upsilon}-2^{1 / 2} i \mathscr{M}^{1 / 2} \varphi \\
& =2^{-1 / 2} \mathscr{M}^{1 / 2}(i \varphi-\mathfrak{S} \varphi-2 i \varphi)=-2^{-1 / 2}(1+i) \mathscr{M}^{1 / 2} \varphi \\
\mathscr{W}(\varphi) & =2^{-1 / 2} i \mathscr{M}^{-1 / 2}(\mathfrak{S} \varphi-i \varphi)=2^{-1 / 2}(1+i) \mathscr{M}^{-1 / 2} \varphi .
\end{aligned}
$$

Нетрудно усмотреть, что ряд $(3.2)$ с коэффициентами $a_{n}=(1+i)\left(\varphi, \varphi_{n}\right)_{\omega}$ сходится в пространстве $\stackrel{\circ}{H}\left(Q ; \partial Q_{+} \backslash \Upsilon\right)$ и экспоненциально затухает на бесконечности, причем согласно определениям $(1.3)_{1}$ и $(1.8)$ сужения $\left.\partial_{z} u\right|_{\Upsilon}$ и $\left.u\right|_{\Upsilon}$ совпадают с выражениями (3.4). Итак, построено продолжение $\mathscr{W}(\varphi)$ в классе $\stackrel{\circ}{H}^{1}(\Omega)$, являющееся (обобщенным) решением задачи (1.1). Осталось повторить конец доказательства леммы 2.1 . 
2. Конечномерные аппроксимации оператора фиктивного рассеяния. Возьмем $\beta>0$ и образуем линейную оболочку $\mathscr{L}_{\bullet}^{\beta}$ тех функций $\varphi_{n}$, для которых $n \in N^{\beta}=\left\{m \in \mathbb{N}:\left|\Lambda_{m}\right|<\beta\right\}:=\left\{1, \ldots, \boldsymbol{n}^{\beta}\right\}$; дополнительно предполагаем, что $\left|\Lambda_{\boldsymbol{n}^{\beta}}\right|<\beta<\left|\Lambda_{1+\boldsymbol{n}^{\beta}}\right|$. Пусть $\mathscr{L}_{\perp}^{\beta}=L_{2}(\omega) \ominus \mathscr{L}_{\bullet}^{\beta}$, а $\mathscr{P}_{\bullet}^{\beta}$ и $\mathscr{P}_{\perp}^{\beta}-$ ортогональные проекторы на подпространства $\mathscr{L}_{\bullet}^{\beta}$ и $\mathscr{L}_{\perp}^{\beta}$ соответственно. Наконец, введем операторы

$$
\mathfrak{S}_{\sigma \tau}^{\beta}=\mathscr{P}_{\sigma}^{\beta} \mathfrak{S} \mathscr{P}_{\tau}^{\beta}, \quad \sigma, \tau \in\{\bullet, \perp\},
$$

а также тождественные отображения $\mathbb{I}$ и $\mathbb{I}_{\sigma}^{\beta}$ в пространстве $L_{2}(\omega)$ и подпространстве $\mathscr{L}_{\sigma}^{\beta}$. Повторение с очевидными изменениями выкладки (3.3) доставляет формулу

$$
\mathfrak{S}_{\bullet \perp}^{\beta} \varphi=0 \quad \forall \varphi \in \operatorname{ker}\left\{\mathfrak{S}_{\perp \perp}^{\beta}-\mathbb{I}_{\perp}^{\beta}\right\} \subset L_{2}(\omega),
$$

а значит,

$$
\mathfrak{S} \varphi=\varphi \quad \forall \varphi \in \operatorname{ker}\left\{\mathfrak{S}_{\perp \perp}^{\beta}-\mathbb{I}_{\perp}^{\beta}\right\} .
$$

Если $\mathfrak{S} \varphi=\varphi$, то $\mathfrak{S}^{*} \varphi=\mathfrak{S}^{-1} \varphi=\varphi$ благодаря унитарности оператора $\mathfrak{S}$, и поэтому по аналогии с формулой (3.6) обнаруживаем, что

$$
\left(\mathfrak{S}_{\perp \bullet}^{\beta}\right)^{*} \varphi=0 \quad \forall \varphi \in \operatorname{ker}\left\{\mathfrak{S}_{\perp \perp}^{\beta}-\mathbb{I}_{\perp}^{\beta}\right\} .
$$

Здесь $\left(\mathfrak{S}_{\perp}^{\beta}\right)^{*}: \mathscr{L}_{\perp}^{\beta} \rightarrow \mathscr{L}_{\bullet}^{\beta}-$ сопряженное отображение. Итак, решая «алгебраическую» систему

$$
\mathfrak{S}_{\bullet \bullet}^{\beta} \varphi_{\bullet}^{\beta}+\mathfrak{S}_{\bullet \perp}^{\beta} \varphi_{\perp}^{\beta}=\varphi_{\bullet}^{\beta}, \quad \mathfrak{S}_{\perp \bullet}^{\beta} \varphi_{\bullet}^{\beta}+\mathfrak{S}_{\perp \perp}^{\beta} \varphi_{\perp}^{\beta}=\varphi_{\perp}^{\beta}
$$

для неизвестных $\varphi_{\bullet}^{\beta}=\mathscr{P}_{\bullet}^{\beta} \varphi$ и $\varphi_{\perp}^{\beta}=\mathscr{P}_{\perp}^{\beta} \varphi$, видим, что ненулевая проекция $\varphi_{\bullet}^{\beta}$ собственной функции $\varphi$ оператора $\mathfrak{S}$, отвечающая собственному числу 1 , обязана удовлетворять равенству

$$
\mathfrak{s}^{\beta} \varphi_{\bullet}^{\beta}=\varphi_{\bullet}^{\beta},
$$

причем

$$
\mathfrak{s}^{\beta}=\mathfrak{S}_{\bullet \bullet}^{\beta}-\mathfrak{S}_{\bullet \perp}^{\beta}\left(\mathfrak{S}_{\perp \perp}^{\beta}-\mathbb{I}_{\perp}^{\beta}\right)^{-1} \mathfrak{S}_{\perp \bullet}^{\beta} .
$$

Поясним определение (3.10): в качестве $\left(\mathfrak{S}_{\perp \perp}^{\beta}-\mathbb{I}_{\perp}^{\beta}\right)^{-1}$ выступает любой линейный обратный, действующий из образа оператора $\mathfrak{S}_{\perp \perp}^{\beta}-\mathbb{I}_{\perp}^{\beta}$ в $\mathscr{L}_{\perp}^{\beta} ;$ при этом в силу (3.8) оператор $\mathfrak{S}_{\perp}^{\beta}$. действует в упомянутый образ и в силу (3.6) левый множитель $\mathfrak{S}_{\bullet \perp}^{\beta}$ устраняет произвол в выборе обратного.

Лемма 3.1. 1) Справедливо равенство

$$
\operatorname{dim} \operatorname{ker}\{\mathfrak{S}-\mathbb{I}\}=\operatorname{dim} \operatorname{ker}\left\{\mathfrak{S}_{\perp \perp}^{\beta}-\mathbb{I}_{\perp}^{\beta}\right\}+\operatorname{dim} \operatorname{ker}\left\{\mathfrak{s}^{\beta}-\mathbb{I}_{\bullet}^{\beta}\right\} .
$$

2) Оператор (3.10) на конечномерном пространстве $\mathscr{L}_{\bullet}^{\beta}$ реализуется как унитарная матрица с элементами $\mathfrak{s}_{p q}^{\beta}=\left(\mathfrak{s}^{\beta} \varphi_{p}, \varphi_{q}\right)_{\omega}$.

ДокАЗАтЕЛЬство. 1) Равенство (3.11), конечномерный аналог которого установлен в $[15,13]$, обеспечено рассуждениями, приведшими к формуле (3.9).

2) Ввиду конечномерности подпространства $\mathscr{L}_{\bullet}^{\beta}$ достаточно проверить изометричность отображения $\mathfrak{s}^{\beta}: \mathscr{L}_{\bullet}^{\beta} \rightarrow \mathscr{L}_{\bullet}^{\beta}$. Для $\psi \bullet \in \mathscr{L}_{\bullet}^{\beta}$ положим $\psi_{\perp}=\left(\mathfrak{S}_{\perp \perp}-\right.$ $\left.\mathbb{I}_{\perp}^{\beta}\right)^{-1} \mathfrak{S}_{\perp \bullet}^{\beta} \varphi_{\bullet} \in \mathscr{L}_{\perp}^{\beta}$ и $\psi=\psi_{\bullet}+\psi_{\perp} \in L_{2}(\omega)$. Нетрудно убедиться в том, что $\mathfrak{S} \psi=\mathfrak{s}^{\beta} \psi_{\bullet}+\psi_{\perp}$, а значит, по теореме 2.1 имеем $\left\|\psi_{\bullet}\right\|^{2}+\left\|\psi_{\perp}\right\|^{2}=\|\mathfrak{S} \psi\|^{2}=$ 
$\left\|\mathfrak{s}^{\beta} \psi_{\bullet}\right\|^{2}+\left\|\psi_{\perp}\right\|^{2}$. Сокращая слева и справа величину $\left\|\psi_{\perp}\right\|^{2}$, получаем нужное равенство.

Формулы (3.7), (3.9) и (3.11) показывают, что для самого оператора фиктивного рассеяния ядро $\operatorname{ker}\{\mathfrak{S}-\mathbb{I}\}$ раскладывается в прямую сумму двух подпространств, причем при малом $\beta>0$ первое подпространство отождествляется с $\operatorname{ker}\left\{\mathfrak{S}_{+}-\mathbb{I}_{+}\right\}$.

СЛЕДСтвиЕ 3.1. Пусть $\beta \in(0, \delta)$, где $\delta>0-$ тот же показатель, что и в (1.5). Тогда оператор $i \mathfrak{s}^{\beta}$ реализуется как матрица рассеяния $s$ и верна формула

$$
\operatorname{dim} \operatorname{ker}\left\{\mathfrak{S}_{+}-\mathbb{I}_{+}\right\}=\operatorname{dim} \operatorname{ker}\{\mathfrak{S}-\mathbb{I}\}-\operatorname{dim} \operatorname{ker}\left\{s+i \mathbb{I}_{0}\right\},
$$

әде $\mathbb{I}_{0}$ - тождественное отображение на компоненте $\mathscr{L}_{0}$ в разложснии (2.2).

ДокАЗАТЕЛьство. Ввиду установленного равенства (3.11) проверить нужно лишь то, что оператор (3.10) реализуется как матрица - is. В силу (2.8) и (2.12), (2.13) решение $w_{p}$ задачи (2.7) (напоминаем, что оно неединственное, но берется какое-нибудь) удовлетворяет краевому условию

$\left.\partial_{z} w_{p}\right|_{\Upsilon}-\left.\mathscr{T} w_{p}\right|_{\Upsilon}=-i\left[2\left(\Lambda_{p}+\chi(p)\right)\right]^{1 / 2} \varphi_{p}+\left.(T-\mathscr{T}) w_{p}\right|_{\Upsilon}=-2^{1 / 2} i \mathscr{M}^{1 / 2}\left(\varphi_{p}+\psi_{p}\right)$, где $\psi_{p}=2^{-1 / 2}(1-i) \mathscr{M}^{1 / 2} \mathscr{P}_{+} w_{p} \mid \Upsilon$. Таким образом, согласно формулам (2.18) и $(2.19)$ справедливы равенства $w_{p}=\mathscr{W}\left(\varphi_{p}+\psi_{p}\right)$ и $\mathfrak{S}\left(\varphi_{p}+\psi_{p}\right)=i\left(\varphi_{p}+\psi_{p}\right)-$ $2^{1 / 2} i \mathscr{M}^{1 / 2} w_{p} \mid \Upsilon$. Проецируя последнее на подпространства $\mathscr{L}_{0}=\mathscr{L}_{\bullet}^{\beta}$ и $\mathscr{L}_{+}=\mathscr{L}_{\perp}^{\beta}$ при учете включений $\psi_{p} \in \mathscr{L}_{+}$и $\left.\mathscr{M}^{1 / 2} \mathscr{P}_{+} w_{p}\right|_{\Upsilon} \in \mathscr{L}_{+}$, в обозначениях (3.5) получаем

$$
\begin{aligned}
\mathfrak{S}_{\bullet \bullet}^{\beta} \varphi_{p}+\mathfrak{S}_{\bullet \perp}^{\beta} \psi_{p} & =i \varphi_{p}-\left.2^{1 / 2} i \mathscr{P}_{0} \mathscr{M}^{1 / 2} w_{p}\right|_{\Upsilon}, \\
\mathfrak{S}_{\perp \bullet \varphi_{p}}^{\beta}+\left(\mathfrak{S}_{\perp \perp}^{\beta}-\mathbb{I}_{\perp}^{\beta}\right) \psi_{p} & =(i-1) \psi_{p}-\left.2^{1 / 2} i \mathscr{P}_{+} \mathscr{M}^{1 / 2} w_{p}\right|_{\Upsilon}=0,
\end{aligned}
$$

а значит, по определению (3.10) $\mathfrak{s}^{\beta} \varphi_{p}=i \varphi_{p}-2^{1 / 2} i \mathscr{P}_{0} \mathscr{M}^{1 / 2} w_{p} \mid \Upsilon$. Интегральные представления (2.10) элементов матрицы рассеяния дают окончательную формулу

$$
\left(\mathfrak{s}^{\beta} \varphi_{p}, \varphi_{q}\right)_{\omega}=i \delta_{p, q}-2^{1 / 2} i\left(\Lambda_{q}+\chi(q)\right)^{1 / 2}\left(\left.w_{p}\right|_{\Upsilon}, \varphi_{q}\right)_{\omega}=-i s_{p q} .
$$

Как упоминалось в пояснениях к соотношениям $(2.6)_{1}$, уходящие волны $U_{1}^{-}, \ldots, U_{n}^{-}$удовлетворяют однородным краевым условиям $(2.7)_{1}$, а значит, и однородным условиям (2.14) благодаря связям (2.13). При $n>\boldsymbol{n}$ затухающие при $z \rightarrow+\infty$ волны $U_{n}^{-}$теряют последнее свойство из-за появления множителя $-i$ при $T \mathscr{P}_{+}$в формуле (2.11). Следуя [14], образуем подобные (1.8) комбинации экспоненциальных волн

$$
\boldsymbol{U}_{n}^{ \pm}=2^{-1 / 2}\left(U_{n}^{-} \mp i U_{n}^{+}\right), \quad n \in N_{-} .
$$

Нетрудно убедиться в том, что функция $\boldsymbol{U}_{n}^{-}$удовлетворяет однородным краевым условиям $(2.14)_{2}$. Назовем волны $\boldsymbol{U}_{n}^{-}$уходящими, а $\boldsymbol{U}_{n}^{+}-$приходящими; в $[13,14]$ на основе такой классификации введена расширенная $\boldsymbol{n}^{\beta} \times \boldsymbol{n}^{\beta}$-матрица рассеяния $s^{\beta}$, составленная из коэффициентов в искусственных условиях излучения

$$
\boldsymbol{u}_{p}(x)=\boldsymbol{U}_{q}^{+}(x)+\sum_{n=1}^{\boldsymbol{n}^{\beta}} \boldsymbol{s}_{p q}^{\beta} \boldsymbol{U}_{q}^{-}(x)+O\left(e^{-z \operatorname{Im} \Lambda_{n^{\beta}}}\right), \quad p=1, \ldots, \boldsymbol{n}^{\beta},
$$


где $\boldsymbol{U}_{m}^{ \pm}=U_{m}^{ \pm}$при $m=1, \ldots, \boldsymbol{n}$, а показатель $\beta$ выбран так, как указано в начале раздела. Матрица $s^{\beta}$ обладает следующим «считающим» свойством [14]:

$$
\operatorname{dim} \mathfrak{L}^{\beta}=\operatorname{dim} \operatorname{ker}\left\{s^{\beta}-\mathbb{I}_{\bullet}^{\beta}\right\}-\operatorname{dim} \operatorname{ker}\left\{s-\mathbb{I}_{0}\right\} .
$$

Здесь $\mathfrak{L}^{\beta} \subset \mathfrak{L}-$ подпространство решений, исчезающих на бесконечности со скоростью не выше $O\left(e^{-z \beta}\right)$. Формулу (3.12) следует интерпретировать как бесконечномерный аналог формулы (3.14).

Несмотря на соотношение $\left.\partial_{z} \boldsymbol{U}_{n}^{-}\right|_{\Upsilon}=\left.\mathscr{T} \boldsymbol{U}_{n}^{-}\right|_{\Upsilon}$ (ср. с комментариями к задаче $(2.4),(2.5))$, говорить о $\mathscr{T}$ как об операторе Стеклова-Пуанкаре для задачи (2.4) с искусственными условиями излучения не приходится, поскольку невозможно придать смысл бесконечным рядам с экспоненциально возрастающими функциями $\left\{\boldsymbol{U}_{n}^{-}\right\}_{n \in N_{-}}$при произвольной $\varphi \in L_{2}(\omega)$, а значит, и найти подходящее продолжение решения $\mathscr{W}(\varphi)$ задачи $(2.14),(2.18)$ на полуцилиндр $Q_{+}$ не удается. Именно поэтому к названию оператора $\mathfrak{S}$, имеющего все свойства обычных операторов рассеяния, добавлен эпитет фиктивный.

Тот факт, что востребованная в настоящей статье вспомогательная задача (2.14) в ограниченной области $G$ однозначно разрешима, тем более удивителен, что попытки $[19,20]$ численной реализации признака $[13,14]$ существования затухающих решений не привели к успеху, в частности, из-за однозначной разрешимости использованной в $[19,20]$ аппроксимационной задачи с искусственными краевыми условиями ${ }^{1)}$ на усекающей поверхности $\Upsilon_{R}=\omega \times\{R\}$. Дело в том, что появление решения однородной задачи в неограниченной области, излишне быстро исчезающего на бесконечности, делает норму обратного оператора недопустимо большой, а погрешности вычислений неприемлемыми. Кроме того, при больших $R$ разность $\boldsymbol{U}_{n}^{+}(y, R)^{-1} \boldsymbol{U}_{n}^{-}(y, R)-1$ составляет $O\left(\exp \left(-2 \Lambda_{n} R\right)\right)$, т. е. на удаленном сечении $\Upsilon_{R}$ волны $\boldsymbol{U}_{n}^{+}(y, R)$ и $\boldsymbol{U}_{n}^{-}(y, R)$ из $(3.13)$ неразличимы для компьютера. При малых $R>0$ искусственное краевое условие $\mathscr{P}_{+} w=0$ на $\Upsilon_{R}$, использованное в $[19,20]$, опять-таки привносит существенные погрешности; при этом схема вычисления расширенной матрицы рассеяния остается устойчивой, но в результате определяется матрица, не имеющая отношения к исходной задаче в области $\Omega$ с волноводом $Q_{+}$.

Задача (2.14), легко поддающаяся численному анализу, лишена упомянутых недостатков прежде всего потому, что теорема 3.1 доставляет критерий существования ловушечных мод, не ставя никаких ограничений на скорость их затухания, а искусственные краевые условия назначены на сечении $\Upsilon=\Upsilon_{0}$, приближенном к резонатору $G$.

Закончим статью конкретным примером решения, быстро исчезающего на бесконечности.

Пример 3.1. Пусть $f$ - гладкая неотрицательная функция, отличная от нулевой, и $\Omega(f)=\left\{x=(y, z) \in \mathbb{R}^{2}:|y|<1 / 2, z>-f(y)\right\}$ - полуполоса с криволинейным основанием $\Sigma(f)$ и боковыми сторонами $\Gamma_{ \pm}(f)$. В работе [21] показано, что при условии

$$
\int_{-1 / 2}^{1 / 2} f(y) \cos (2 \pi y) d y>0
$$

${ }^{1)} \mathrm{B}[19,20]$ эти условия включают равенство $\mathscr{P}_{\perp}^{\beta} w=0$ на $\Upsilon_{R}$, демпфирующее волны, которые затухают со скоростью $o(\exp (-\beta z))$. 
найдутся волновое число $k_{1} \in(0, \pi)$ и затухающее как $O\left(\exp \left(-z\left[\pi^{2}-k_{1}^{2}\right]^{1 / 2}\right)\right)$ нетривиальное решение $u_{1}$ смешанной краевой задачи

$$
\begin{gathered}
\Delta u(x)+k^{2} u(x)=0, \quad x \in \Omega(f), \\
u(x)=0, \quad x \in \Gamma_{ \pm}(f), \quad \partial_{\nu} u(x)=0, \quad x \in \Sigma(f) .
\end{gathered}
$$

Обращаем внимание на то, что лишь для упрощения изложения ранее граница $\partial \Omega$ предполагалась гладкой и всюду на ней ставились условия Дирихле, - возможные сингулярности в угловых точках и точках смены типа краевого условия не выводят решение из соболевского класса $H^{1}$.

Пусть $f(y)=0$ при $|y| \in(1 / 4,1 / 2)$ и $f(0)>0$, т. е. ограничение (3.15) выполнено. Продолжим функцию $f$ по 1 -периодичности на ось $\mathbb{R}$ и положим $f_{m}(y)=$ $m^{-1} f\left(m^{-1} y\right)$, где $m \in \mathbb{N}$ и $m>1$. Благодаря условиям Дирихле в задаче (3.16) решение $u_{1}$ продолжается по нечетности через лучи $\{x: y=j+1 / 2, z>0\}$, $j \in \mathbb{Z}$, до гладкой 2 -периодической функции на надграфике функции $f$. Поэтому $u_{m}(x)=u_{1}\left(m^{-1} x\right)$ - решение задачи (3.16) в области $\Omega\left(f_{m}\right)$ с волновым числом $k=m k_{1}$. Это решение убывает со скоростью $O\left(\exp \left(-z m\left[\pi^{2}-k_{1}^{2}\right]^{1 / 2}\right)\right)$ и поэтому в его разложении отсутствуют волны

$$
U_{n}^{-}(x)=c_{n} \exp \left(-z\left[\pi^{2} n^{2}-k_{m}^{2}\right]^{1 / 2}\right) \sin (\pi n(y+1 / 2)),
$$

где $n=1, \ldots, m-1$, а $c_{n}$ - нормирующий множитель. Нетрудно видеть, что в случае $n \in\left(m k_{1} \pi^{-1}, m\right)$ волны (3.17) экспоненциально затухают при $z \rightarrow+\infty$, причем за счет увеличения коэффициента $m$ количество таких волн можно сделать сколь угодно большим.

\section{Литература}

1. Wilcox C. Scattering Theory for Diffraction Gratings. Springer-Verlag, Berlin, 1980.

2. Назаров С. А., Пламеневский Б. А. Принципы излучения для самосопряженных эллиптических задач. В сб.: Дифференциальные уравнения. Спектральная теория. Проблемы матем. физики, вып. 13, Изд-во ЛГУ, Л., 1991, с. 192-244.

3. Назаров С. А., Пламеневский Б. А. Эллиптические задачи в областях с кусочно гладкой границей. Наука, М., 1991.

4. Ursell F. Trapping modes in the theory of surface waves. Proc. Cambridge Philos. Soc., 47, 347-358 (1951).

5. Jones D. S. The eigenvalues of $\nabla^{2} u+\lambda u=0$ when the boundary conditions are given on semi-infinite domains. Proc. Cambridge Philos. Soc., 49, 668-684 (1953).

6. Evans D. V. Trapped acoustic modes. IMA J. Appl. Math., 49, 45-60 (1992).

7. Linton C. M., Evans D. V. Integral equations for a class of problems containing obstacles in waveguides. J. Fluid Mech., 245, 349-365 (1992).

8. Bonnet-Bendhia A.-S., Starling F. Guided waves by electromagnetic gratings and nonuniqueness examples for the diffraction problem. Math. Methods Appl. Sci., 17, No. 5, 305-338 (1994).

9. Evans D. V., Levitin M., Vasil'ev D. Existence theorems for trapped modes. J. Fluid Mech., 261, 21-31 (1994).

10. Готлиб В. Ю. О решениях уравнения Гельмгольца, сосредоточенных вблизи плоской периодической границы. Зап. научн. семин. ПОМИ, 250, 83-96 (1998).

11. Сухинин C. В. Волноводное, аномальное и шепчущее свойства периодической цепочки препятствий. Сиб. журн. индустр. матем., 1, №2, 175-198 (1998).

12. Камоцкий И. В., Назаров С. А. Экспоненциально затухающие решения задачи о дифракции на жесткой периодической решетке. Матем. заметки, 73, № 1, 138-140 (2003). 
13. Камоцкий И. В., Назаров С. А. Аномалии Вуда и поверхностные волны в задачах рассеяния на периодической границе. 1. Матем. сб., 190, №1, 109-138 (1999); 2. ibid., 190, № 2, 43-70 (1999).

14. Камоцкиц̆ И. В., Назаров С. А. Расширенная матрица рассеяния и экспоненциально затухающие решения эллиптической задачи в цилиндрической области. Зап. научн. семин. ПОМИ, 264, 66-82 (2000).

15. Назаров C. А., Пламеневский Б. А. Самосопряженные эллиптические задачи: операторы рассеяния и поляризации на ребрах границы. Алгебра и анализ, 6, № 4, 157-186 (1994).

16. Лионс Ж.-Л., Мадженес Э. Неоднородные граничные задачи и их приложения. Мир, М., 1971.

17. Trudinger G. Elliptic Partial Differential Equations of Second Order. Springer-Verlag, Berlin, 1983.

18. Лифиии, M. С. Операторы, колебания, волны (Открытые системы). Наука, М., 1966.

19. Грикуров В. Э., Нейтаамяки П., Пламеневский Б. А., Хейккола Е. О методе поиска поверхностных волн в дифракционных решетках. Докл. РАН, 385, №4, 465-469 (2002).

20. Grikurov V. E., Heikkola E., Niettaanmäki P., Plamenevskii B. A. On computation of scattering matrices and on surface waves for diffraction gratings. Numer. Math., 94, 269-288 (2003).

21. Камоцкий И. В., Назаров С. А. О собственных функциях, локализованных около кромки тонкой области. В сб.: Проблемы матем. анализа. Вып. 19, Научная книга, Новосибирск, 1999, с. 105-148.

Институт проблем машиноведения РАН

email: serna@snark.ipme.ru

Поступило в редакцию 21 декабря 2004 г. 